\title{
Measurement of Root Hydraulic Conductance
}

\author{
Albert H. Markhart, III \\ Department of Horticulture Science and Landscape Architecture, University of Minnesota, \\ St. Paul, MN 55108 \\ Barbara Smit \\ Center for Urban Horticulture, University of Washington, Seattle, WA 98195
}

Plant root systems provide water, nutrients, and growth regulators to the shoot. Growth and production of a plant are often limited by the ability of the root to extract water and nutrients from the soil and transport them to the shoot. The transport of most nutrients and growth regulators occurs via the transpiration stream. The velocity and quantity of water moving from the root to the shoot determines the quantity and concentration of substances that arrive at the shoot. Understanding the forces and resistances that control the movement of water through the soil-plant-air continuum and the flux of potential chemical signals is essential to understanding the impact of the soil environment on root function and on root integration with the shoot.

The objectives of this paper are: a) to discuss the nature of the resistances that limit water flux through the root, b) evaluate methods of measuring root hydraulic conductance, c) describe the effect of temperature and oxygen on root function, and d) discuss the nature of the signals that may be sent from the root to the shoot.

\section{General principles and definition of terms}

Root systems are the rate-limiting site for water movement from the soil to the leaf (Kramer, 1983). This fact is easily demonstrated by taking two wilted tomato plants, placing both in a beaker of water, and excising the root system of one while leaving the other intact. The plant without a root system will regain turgor more rapidly.

Root resistance to water flow is substantial enough to cause low leaf water potentials, even under well-watered conditions. Water potentials of hydroponically grown soybean plants in a growth chamber can reach -0.7 MPa (Markhart et al., 1980). Midday leaf water potentials in well-watered fields can get low enough to cause stomatal closure and reduce photosynthesis.

We are concerned in this paper with water moving from the rootsoil interface to the apoplast of the leaves. Out of necessity, we will divide this pathway into two components. The radial component is from the root-soil interface to the root xylem. The axial component is from the root xylem to the apoplast of the leaf. The flux $(\mathrm{Q})$ through each pathway is determined by the water potential gradient $\left(\Delta \psi_{w}\right)$ across the pathway and the conductance (L) of the pathway. If the surface area is known, then the hydraulic conductance can be converted to a hydraulic conductivity (Lp) by dividing $\mathrm{L}$ by the surface area.

$$
\mathrm{Q}=\Delta \psi_{\mathrm{w}} \cdot \mathrm{L}
$$

The water potential gradient is the difference between the water potential of the soil at the root surface and the water potential of the water in the root xylem. The soil water potential is the sum of the matric potential and the osmotic potential of the soil solution. This quantity is relatively easy to measure. The water potential of the water in the xylem is a different matter. The $\psi_{w}$ of water in the xylem is the sum of the water potential generated by the pull from

Published as paper 16993 of scientific journal series of the Minnesota Experiment Station on research conducted under Minnesota Experiment Station Project 0302-4821-82. Additional support was provided by the Univ. of Washington Graduate School Research Fund, Horticultural Research Institute Grant. transpiration at the shoot and osmotic potential. The latter is generated by the combination of active solute accumulation, passive solute leakage, and rate of water movement from the soil to the xylem. This is difficult, if not impossible, to determine. Conductivity of the radial pathway is determined by structures through which the water flows. Water flows along the path of least resistance. Resistance of the interstices in the cell wall is considered lower than across plasmalemma and cytoplasm. For these reasons, it is thought that water moves apoplasticly across the root until a significant barrier is encountered, at which point the water is forced through the plasmalemma. This apoplastic barrier is the suberinimpregnated endodermal or hypodermal cell wall. Recently, apoplastic movement across the root has been questioned (Steudle and Jeschke, 1983). Using the pressure probe measurements of individual cell Lp and root conductance, they argued that the water moves from cell to cell symplastically and not through the apoplast. Regardless of the exact pathway across the root, at some point, water passes through a highly semipermeable barrier that has characteristics very much like a plasmalemma. In healthy root systems, only $1 \%$ of the water either leaks through or bypasses the semipermeability barrier (Hanson et al., 1985).

The casparian band was initially described at the endodermis, but recently has also been described in many plant species as part of a hypodermis located one or two cell layers in from the epidermis (Peterson, 1988). The physical location of the rate-limiting barrier is of considerable interest because it determines where the major potential drop occurs in the transport pathway, and, therefore, the water potential of the different root tissues. For example, if the endodermis is the rate-limiting barrier, then the water potential of the root cortex is close to the water potential of the soil. If, however, the rate-limiting barrier is at the hypodermis, then the water potential of the cortex will be closer to that of the shoot, and, during high transpiration rates, will be much lower than the soil. This aspect could be critical when considering the part of the root sensing the environment and sending chemical messages to the shoot.

The location of the rate-limiting barrier is also important when considering the root-soil interface. A root with an endodermal barrier would undergo less shrinkage during times of high transpiration rate or water deficit than roots with a hypodermal barrier (Passioura, 1988). If roots do shrink under field conditions, a major resistance could occur at the root-soil interface due to poor contact between the root and the liquid phase of the soil solution.

Another important characteristic of the pathway is the ability to accumulate ions actively. At low transpiration rates, this generates an osmotic gradient, driving water into the stele, which is the source of root pressure and guttation. Although we prefer to think of the radial pathway of water movement as a simple pathway, it is important to realize that the pathway is complex, with highly selective barriers and active pumps, with metabolizing cells interacting with a solution pulled through them at varying velocities.

The axial or longitudinal pathway of dead xylem elements connecting the root and the shoot is deceptively simple. Although the conducting elements themselves are dead tubes, they abut xylem parenchyma cells that are metabolically active. In fact, there have been reports of transfer cell-like membrane invaginations (Lauchli, 1976). Whether these cells pump solutes into the xylem or out, or both, is unclear. In any event, it is clear that the concentration and composition of the xylem sap change as it moves up the root. 


\section{Methods of measuring conductance}

General. The general principle of measuring root conductance is similar to the measurement of membrane permeability. In a membrane, a chemical potential gradient of the substance of interest is established across the membrane and the flux of the substance is measured. Then, using an equation analogous to Eq. [1], conductance of the membrane is calculated. Conductivity or conductance per unit area can be calculated if the surface area of the membrane is known. Hydraulic conductivity of a root system is measured in a similar fashion. A known water potential difference is established across the root and the water flux through the root system measured. The procedure at first glance is relatively simple. The simplicity, however, stems from a number of assumptions that, in fact, are rarely met. These have been discussed in considerable detail by Fiscus (1975), Dalton et al. (1975), and Newman (1976), but deserve a less quantitative description here.

Water transport is relatively easily measured in roots. Water lost from a reservoir, weight lost from a pot, and transpiration rate measured by gas exchange techniques all work well with whole plants. Detopped root systems give the advantage of being able to measure the flow of exudate from the cut stump.

The major problem is the inability to know adequately the water potential gradient across the membrane or barrier that is limiting water flux. Since the total water potential is composed of a hydrostatic component and an osmotic component, we must know, or be able to estimate reasonably, the hydrostatic potential component and the solute potential on each side of the limiting barrier. The hydrostatic component is straightforward, but the solute potentials are a major problem.

The usual assumption is that the solute potential, acting at the external surface of the limiting barrier, is the same as the external solution and that the solute potential inside the barrier is the same as the exudate emerging from the cut surface of the stump. Unfortunately, neither of these assumptions is very good. Fiscus (1975) has pointed out that, because of the ultrafiltration characteristics of the root, the concentration of solutes at high flow rates at the effective barrier would be significantly higher than that of the ambient solution and the concentration could be less than the ambient at low flow rates if the rate of active transport were greater than the rate of diffusion from the ambient solution to the limiting barrier (Fig. 1).

The concentration inside the limiting barrier also changes considerably with flow due to the combined effects of ultrafiltration and active transport (Fig. 1; see Fig. 3). In addition, the concentration changes as the xylem sap moves toward the shoot (Anderson
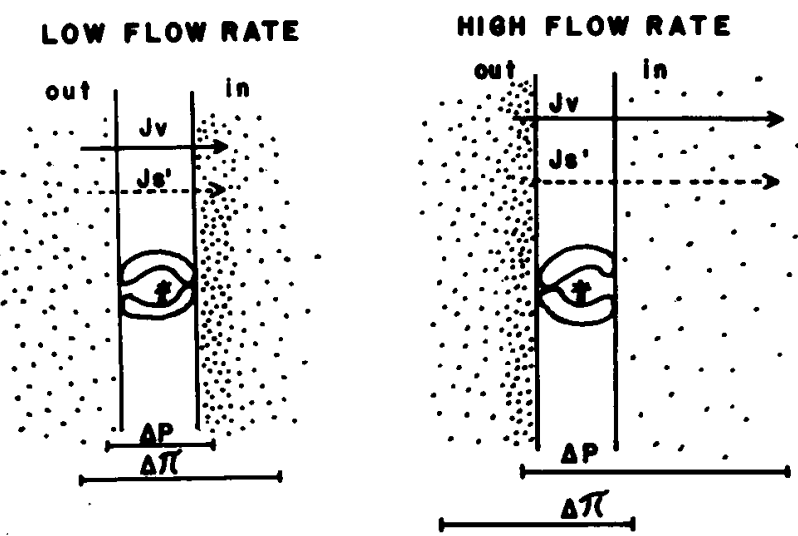

Fig. 1. Large and small hydrostatic potential gradient effects on water flux and osmotic potentials across a semipermeable barrier containing an active solute pump. $\mathrm{Jv}=$ total volume flux, Js' = salt flux, $\Delta \mathrm{P}=$ hydrostatic pressure gradient, and $\Delta \pi=$ osmotic potential gradient. At low transpiration rates the active accumulation of ions (Js') generates an osmotic potential gradient that pulls water into the root. At high transpiration rates, ultrafiltration of the semipermeable barrier results in dilution of the xylem sap in the root and, potentially, an accumulation of solutes outside the barrier. The result is an osmotic gradient that drives water out of the root toward the ambient solution. et al., 1970; Klepper, 1967). Basal portions of the roots of many species actively extract ions from the transpiration stream and either sequester them in the cortex or pump them back out into the ambient solution. In other words, what one measures in the stem xylem is not what is osmotically effective at the osmotically active barrier in the root.

\section{Implications for measuring $\mathrm{Lp}$}

The interaction of hydrostatic potential and osmotic potential is most severe at low fluxes. The osmotic potential gradient decreases as the hydrostatic potential gradient increases, resulting in little net change in total water potential driving water movement through a root system. This would not be a problem if one could determine the internal and external osmotic potential and calculate the total water potential gradient accurately. This is, however, impossible. This situation suggests the measurements of Lp using root pressuregenerated flow and varying the external solution water potential by the addition of osmoticum are flawed because of the inability to measure the water potential gradient accurately.

The same problem exists for measurements that involve suction applied to the cut stem. The maximum hydrostatic pressure gradient produced by pulling a vacuum is $\approx 70 \mathrm{kPa}$. This is precisely the range where the interaction of hydrostatic and osmotic driving forces is most pronounced. In other words, a step change in hydrostatic potential gradient results in a smaller-than-expected total water potential change because the osmotic potential changes in the opposite direction to the hydrostatic potential. This problem explains the often-observed curvilinear relationship between hydrostatic potential gradients and flux at low hydrostatic potential gradients.

If the osmotic potential gradient changes substantially relative to the hydrostatic potential gradient and is impossible to measure, then accurate measurements of Lp can only be made over steps in hydrostatic potential where the osmotic potential gradient changes very little. In most root systems this occurs at a hydrostatic potential gradient $>300 \mathrm{kPa}$. As indicated in Fig. 2, the osmotic potential of the exudate changes very little at this flux, and the flux vs.

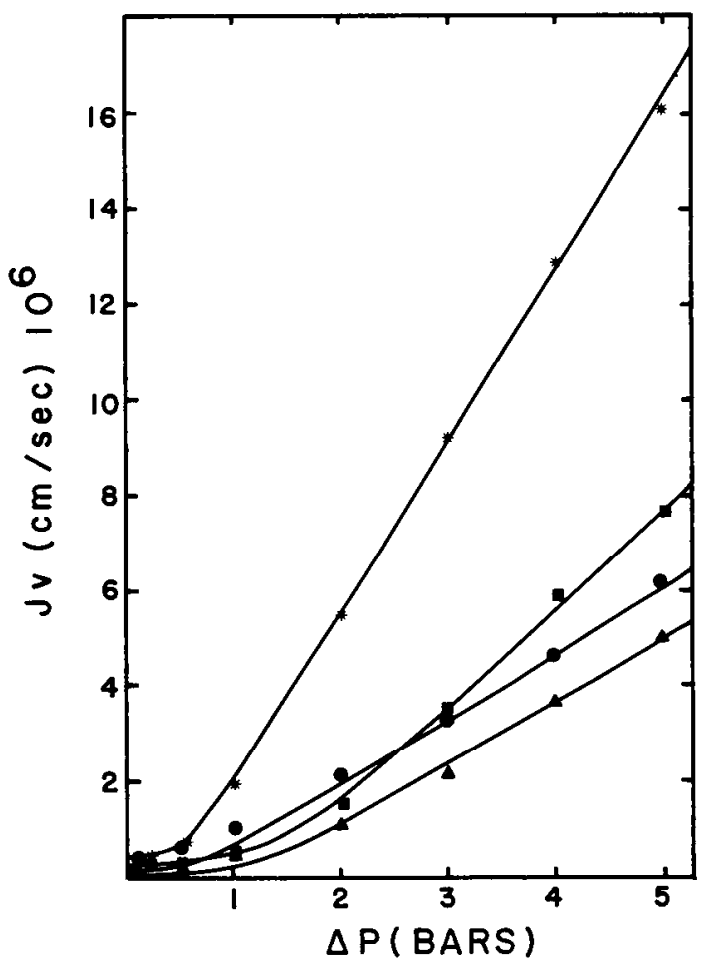

Fig. 2. Relationship between flow rate (Jv) and applied pressure $(\Delta \mathrm{P})$ for de-topped root systems grown and measured in half-strength Hoagland's solution at $25 \mathrm{C}$. Water flux is expressed per unit of root length. $(*)$ soybean $\mathrm{Lp}=3.6 \mathrm{E}-6 \mathrm{~cm} \cdot \mathrm{s}^{-1} \cdot \mathrm{bar}^{-1},(\boldsymbol{)})$ tomato $\mathrm{Lp}=1.37 \mathrm{E}-6$ $\mathrm{cm} \cdot \mathrm{s}^{-1} \cdot \mathrm{bar}^{-1},(\boldsymbol{\Delta})$ sunflower $\mathrm{Lp}=1.3 \mathrm{E}-6 \mathrm{~cm} \cdot \mathrm{s}^{-1} \cdot \mathrm{bar}^{-1}$, and $(\boldsymbol{\square})$ broccoli $\mathrm{Lp}=2.1 \mathrm{E}-6 \mathrm{~cm} \cdot \mathrm{s}^{-1} \cdot \mathrm{bar}^{-1} .(1 \mathrm{bar}=100 \mathrm{kPa})$. 
hydrostatic potential relationship is linear (Fig. 3). The slope of this relationship is a good measure of the conductance of the root system.

\section{Criticisms of de-topped root systems exposed to large hydrostatic potential}

Unnatural paths. The use of de-topped root systems exposed to large hydrostatic potential has been criticized because of the possibility that positive pressure forces solution up the root through apoplastic paths in the cortex that water does not naturally follow when pulled by transpiration (Salim and Pitman, 1984; DeBoer and Prins, 1984). Another possibility is that flow could be induced in the phloem, which would increase the apparent exudation rate at the cut surface and decrease the osmotic potential by the addition of solutes. For these reasons, care must be taken to remove the bark and cortical tissue of the stem below the cut surface and seal the exposed phloem adequately. Adequate seals are necessary to prevent spurious flow of nutrient solution up fissures in the bark or directly through the seal. The seal can be tested by regularly measuring the osmotic potential of the exudate at high flow rates. The exudate osmotic potential from the species is 5 to $10 \mathrm{kPa}$ when the ambient solution was half-strength Hoagland's solution $\left(\psi_{\mathrm{s}}=50\right.$ $\mathrm{kPa}$ ). We also have used an apoplastic marker (Hanson et al., 1985) and found $<1 \%$ of the water moved exclusively by an apoplastic pathway. Similarly, food dye added to the bathing solution appears in the xylem exudate from injured roots, but not in the exudate of healthy roots (Smit and Stachowiak, 1988).

Root perfusion. Root systems that are placed under pressure for several hours often appeared water-soaked and translucent. The lack of air-filled spaces has raised the question that roots may suffer from anoxic conditions that would affect the transport characteristics. In our own work, we were more concerned with too high $\mathrm{O}_{2}$ tensions produced by the effects of pressure on the amount of gas dissolved in the solution (A.H.M. and E. Fiscus, unpublished data). If compressed breathing air is used to generate the hydrostatic potential gradient, the $\mathrm{O}_{2}$ tension increases proportionally to the applied pressure. Applied pressures of $500 \mathrm{kPa}$ would raise the $\mathrm{O}_{2}$ tension in the ambient solution to $100 \mathrm{kPa} \mathrm{O}_{2}$. To determine if these

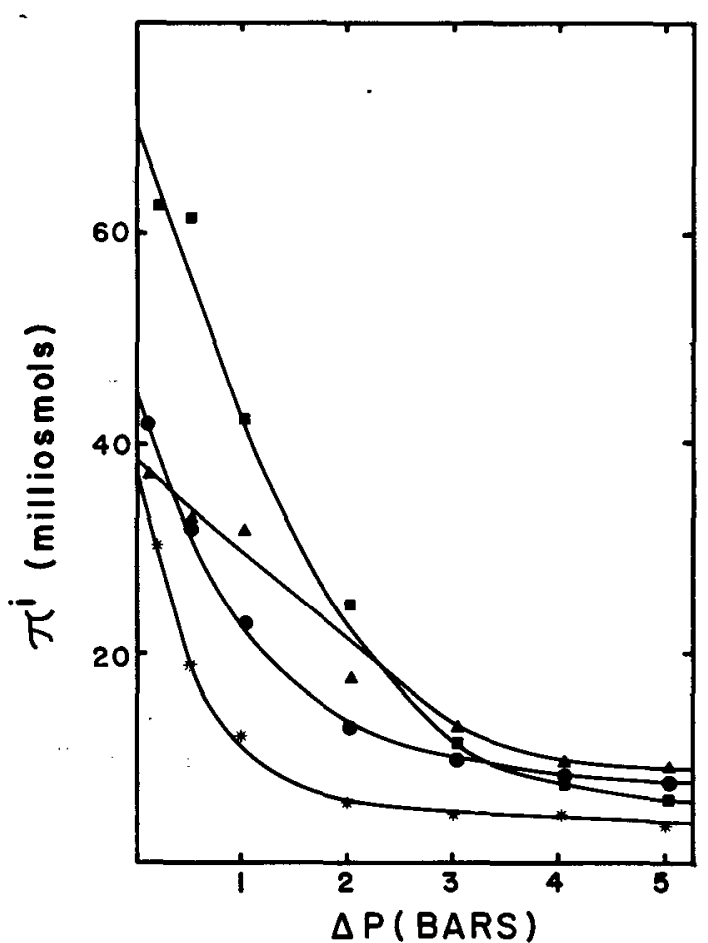

Fig. 3. Exudate osmolality vs. applied hydrostatic pressure for de-topped root systems grown and measured in half-strength Hoagland's solution at 25C. The osmolality of the ambient solution was $22 \mathrm{mmol}$. (*) Soybean, ( $)$ sunflower, $(\boldsymbol{\Delta})$ tomato, and $(\boldsymbol{\square})$ broccoli. $(1 \mathrm{bar}=100 \mathrm{kPa})$. high tensions were damaging to roots, we varied the $\mathrm{O}_{2}$ concentration of the gas used to pressurize the roots so that the total oxygen tension of the pressurized solution was $21 \mathrm{kPa}$ at each pressure. Water flux vs. applied pressure curves from roots treated with constant $21 \mathrm{kPa} \mathrm{O}_{2}$ were not different from curves generated with increased $\mathrm{O}_{2}$ tensions. We concluded from these results that high$\mathrm{O}_{2}$ did not affect water flux.

Lack of a shoot. Another major criticism of de-topped root experiments is the lack of a continued supply of photosynthate and chemical messages to the root. Removal of phloem transport from shoot to root affects ion uptake (Bowling et al., 1985), and detopping may therefore affect osmotically driven water uptake. Water transport at relatively high flow rates, however, does not appear to be affected. Two flux vs. hydrostatic potential curves were generated with the same root system, one immediately after excision from the shoot and the second the next day. The root system remained in the pressure chamber between the two pressure series and was constantly aerated (Fig. 4). Note that the slopes of the flux vs. pressure curves at high pressures are not different, indicating that the Lp of the root did not change during $24 \mathrm{hr}$. The flux at low pressure $(<100 \mathrm{kPa})$ on the 2 nd day, however, was only half the flux on the first day. The solute concentration on the 2 nd day was also $40 \%$ lower than on the first. These results encourage the use of de-topped root systems for measurements of root Lp at high flow rates; however, they question the appropriateness of their use for measurement of solute uptake and transport.

\section{Precautions of using hydrostatic potential on de-topped roots}

In addition to adequate sealing and removal of the bark as outlined above, there are several other aspects of the technique that must be considered.

Multiple pressures. Too often, investigators have used the flux measured at a single applied pressure to calculate the $\mathrm{Lp}$ of the root. The assumption that is made in this calculation is that flux at zero applied pressure is zero. This assumption is not true (Fig. 2), and has been discussed in detail elsewhere (Fiscus, 1975; Dalton et al.,

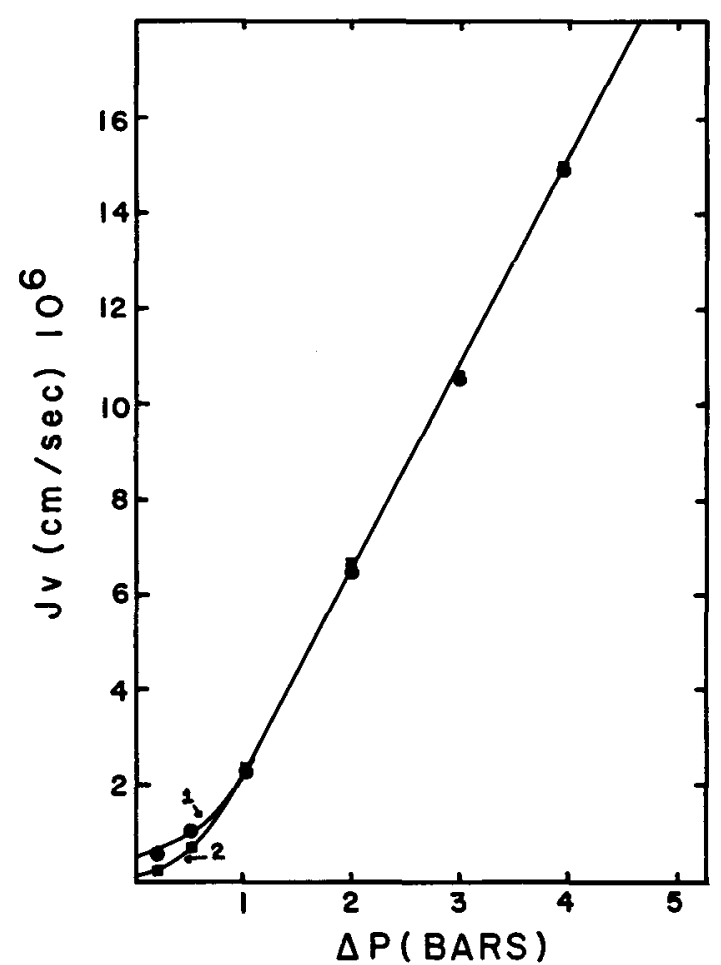

Fig. 4. Time effects on flow rate and exudate concentration. The curve labeled " 1 " ( ) was generated the first day after shoot excision. The curve labeled "2" ( $\mathbf{\square})$ was generated after the root system was left with $20 \mathrm{kPa}$ of applied pressure overnight. Plants were soybeans grown in halfstrength Hoagland's solution at 29/23C thermoperiod and measured at 25C. $(1 \mathrm{bar}=100 \mathrm{kPa})$. 
1975; Passioura, 1988). The error created by using a single pressure can be as high as $30 \%$ (Fig. 2). It is therefore important to measure flux at several applied pressures. We regularly use measurements at 300,400 , and $500 \mathrm{kPa}$ as a minimum, with linear regression analysisto calculate the slope of the line. The experiment is rejected if the regression coefficient is $<0.95$.

Steady states. An important assumption in using measured fluxes and applied pressures to calculate a conductance is that the fluxes and potentials are not changing with time, that steady-state conditions exist. This is not as straightforward as it initially appears. After the pressure is initially applied, water flux increases for several hours until a steady state is reached. The subsequent pressure steps reached steady states much more rapidly. We consider the system is at steady state if the water flux and solute concentration of the exudate do not change more than $1 \%$ during $15 \mathrm{~min}$.

Diurnal rhythms. Diurnal rhythms in Lp and solute pumping have been described in roots (Fiscus, 1986; Parsons and Kramer, 1974). This can pose serious problems to measurements of $\mathrm{Lp}$. In our own work, we find amplitudes of the rhythms are larger at low pressures than at high pressures. In soybean and common bean, where these diurnal rhythms have been measured extensively, we find little diurnal fluctuation in water flux at constant pressures $>400 \mathrm{kPa}$. However, as a precaution, we start all our experiments at the same time of day, usually 8:00 AM, at the beginning of what would be the light cycle, let the root systems adjust for $4 \mathrm{hr}$ at the first pressure, and then begin the pressure series. Each. pressure usually takes between 0.5 and $1 \mathrm{hr}$ to complete.

Ambient solution. The ambient solution used for making measurements of Lp is important. We have grown plants in half-strength Hoagland's solution or in a loose soil mix of sand and surface and then measured the root Lp in half-strength aerated Hoagland's solution. The solution should be the same in which the plants were grown or acclimated prior to the measurement. A number of $\mathrm{Lp}$ measurements have been made by placing the roots in distilled water for the measurement. This shock can have serious effects on the transport characteristics of the root. Osmotic relations and nutrient pumps will affect the osmotic potential component of the driving force. In addition, leaching of calcium from the root cortex is known to affect plasmalemma permeability seriously. Minimizing the shock between growth and measurement conditions will increase accuracy and repeatability of Lp measurements.

Some plants have very fragile roots that easily are injured during transfer from pot to solution culture. When roots are injured, it is not possible to get steady-state flux through the roots (Smit and Stachowiak, 1988). These species must be pre-climated to solution culture before excision and measurement. Finally, measurements can be made on potted roots in well-watered media. This is useful for mycorrhizal root systems or other cases where roots cannot be disturbed (Andersen et al., 1988). If the medium is well-watered, the resistance to flow through the medium is minimal and can be ignored.

Aeration. Aeration of the ambient nutrient solution is critical to the health of the root system. Continued flow through the solution not only ensures adequate gas exchange, but also aids in mixing the solution. A Scholander-type pressure bomb filled with nutrient solution to measure root $\mathrm{Lp}$ has been tried in some investigations. The respiration of roots and bacteria rapidly lower the $\mathrm{O}_{2}$ in the ambient solution without the continued flow of air through it. Hanson and co-workers found that red pine root systems lower dissolved $\mathrm{O}_{2}$ to injurious levels within $4 \mathrm{hr}$ (Hanson et al., 1985). The worst case is when the $\mathrm{N}_{2}$ tank is left attached to the pressure bomb and used to pressurize the root system.

Basis for converting conductance to conductivity. The basis for comparing root systems of different sizes, treatments, and species requires the choice of some characteristic to normalize the total flow of water through the root system. The ideal measure is the surface area of the rate-limiting barrier in the root, but its identity and, therefore, dimensions are unknown. Various root and shoot characteristics have been used by different investigators. Root surface area, although difficult to measure, is often used, as are root length and root dry or fresh weight. The usual assumption is that these other characteristics vary linearly with surface area and are, there- fore, useful comparisons. This approach is valid for plants of the same genotype treated similarly, but may be questionable if plants with distinctly different root morphology are compared. The other assumption is that all the roots have the same hydraulic conductivity. This is, of course, not correct, but the error introduced is, one hopes, the same in the root systems being compared. The assumption breaks down when the number of new root tips to mature root tips varies. Fiscus and Markhart (1979), for example, showed that the average Lp of soybean roots changed substantially with age between 15 and 35 days. These changes were correlated with secondary root development. Root tips are considered the major site of water uptake; hence, several investigators have expressed flow rates on a root tip basis (Smit-Spinks et al., 1984). This is feasible for smaller root systems, but not realistic for, root systems from mature plants.

\section{Environmental effects on root Lp}

A membrane is the likely limitation to water flux through root systems; thus, it is not surprising that environmental conditions that affect permeability characteristics of membranes affect water absorption through root systems significantly. Temperature, $\mathrm{O}_{2}$ levels, salt levels, and nutrient concentrations have been shown to affect water flux through the root system and root hydraulic conductance. As the discussion above has pointed out, conclusions based on changes in water flux must be made cautiously. An important example is the effect of increased salt concentrations. Increased levels of sodium chloride or potassium chloride cause a rapid decrease in water flux through the root system. The decrease is due to a change in the water potential gradient across the root system because of the decreased soil osmotic potential. The change in flux gives no information on the effect of the change in salt concentration on root hydraulic conductance. Water flux over a range of hydrostatic pressure potentials must be determined to ascertain if the root hydraulic conductance is also affected. Short-term studies on a single root system show that low levels of osmoticum affect water flux, but do not affect the root hydraulic conductance (Markhart, 1978; Shalhevet et al., 1976). Others have found that long-term exposure to sodium chloride does affect the hydraulic conductance of the root system, which contributes to water stress in the shoot (O'Leary, 1969; Choukrallah and Markhart, unpublished data).

Temperature. Root systems are also highly sensitive to temperature. Low soil temperature reduces root conductance in many species. Chilling-sensitive species often wilt if watered with cold water at a time of high transpirational demand. Of particular interest is the capability of the root system to acclimate to decreased temperatures. In earlier work (Markhart et al., 1979), we found that soybeans grown at decreased temperatures had a significantly lower "break point", or critical temperature at which root system conductance dramatically decreased. This was also correlated with a decrease in the degree of saturation of the bulk root fatty acid unsaturation (Markhart et al., 1980). Likewise, the low-temperature threshold of roots of different species may influence plant distribution (Teskey et al., 1984).

More recently, we found that roots acclimate rapidly to a temperature change (Markhart, 1987). The hydraulic conductance decreases to a minimum $\approx 1 \mathrm{hr}$ after the temperature decrease if the temperature of de-topped root systems exposed to a $300-\mathrm{kPa}$ hydrostatic potential gradient is decreased by $15 \mathrm{C}$. The flux of water recovers substantially after $2 \mathrm{hr}$ at the low temperature. Recovery of the root system correlates with opening of stomata in intact plants with chilled root systems. The ability of the root system to continually adjust as the root grows into new environments, or as the environment changes, is of considerable adaptive advantage to plants growing in a heterogeneous or fluctuating environment.

Hypoxia. Soil flooding and compaction result in hypoxic or anoxic conditions (Smit and Stachowiak, 1988). The low- $\mathrm{O}_{2}$ and high$\mathrm{CO}_{2}$ conditions have significant effects on root hydraulic conductivity (Fig. 5). Interestingly, exposure to $100 \% \mathrm{~N}_{2}$ (Fig. 5b) (or $20 \%$ $\mathrm{CO}_{2}$ with or without $20 \% \mathrm{O}_{2}$ (Fig. $5 \mathrm{c}$ and d), caused a rapid decrease in root hydraulic conductivity. The decrease in the presence of $\mathrm{CO}_{2}$ was not related to the decrease in ambient solution $\mathrm{pH}$, 


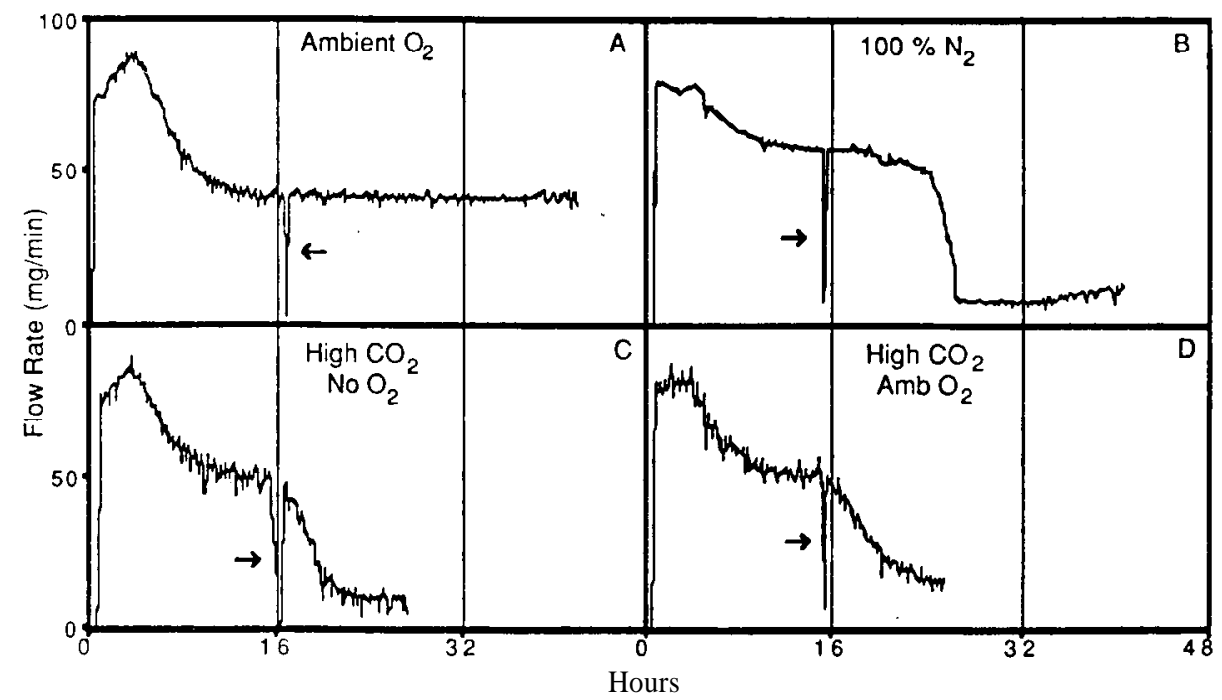

Fig. 5. Representative traces of xylem sap flow rate from poplar root systems treated with (A) ambient air; (B) $100 \% \mathrm{~N}_{2} ;\left(\right.$ C) $20 \% \mathrm{CO}_{2}, 80 \% \mathrm{~N}_{2} ;$ (D)

$20 \% \mathrm{CO}_{2}, 20 \% \mathrm{O}_{2}, 60 \% \mathrm{~N}_{2}$. Arrow indicates the point at which the pressure vessel was depressurized and repressurized with the treatment gas.

which dropped from 6 to 4.2 when aerated with $20 \% \mathrm{CO}_{2}$. The mechanism of the decline in hydraulic conductivity due to $\mathrm{CO}$, is not known.

\section{Root to shoot communication}

Root systems transport water, nutrients, and organic compounds to the shoot. Suboptimal root-zone conditions decrease water or nutrient flux, which, in turn, reduces shoot growth. The effect of the root on the shoot in this situation is essentially as a passive supplier. The only time the shoot experiences a problem is when the supply of a needed resource is reduced. One can imagine an alternative condition where the root sends a signal to the shoot indicating that a resource is declining and may become limiting. This signal would enable the shoot to take preventive measures so that injury would not be severe.

An interesting example is the case of a slowly drying soil. Traditional water relations have taught that the water status of the soil is communicated to the shoot by a purely hydraulic signal. The shoot water potential decreases to a point where stomata close and photosynthesis is inhibited as the soil water potential decreases. Recent evidence suggests that signals other than just hydraulic signals communicate the soil water status to the shoot (Davies et al., 1986; Gollan et al., 1986). The two principal candidates are abscisic acid and cytokinins. Cytokinins are synthesized in root tips and prevent senescence and keep stomata open. Abscisic acid causes stomatal closure and is produced in roots subjected to osmotic stress (Walton et al., 1976; Baker and Lachno, 1987). Most of the recent evidence supports such a role for ABA, while cytokinin responses are less clear. The sensitive leaf tissue can respond by closing stomata and reducing growth, before the leaf water potential decreases to damaging levels. This connection could explain observations of low stomata1 conductances in stressed plants that have high turgor (Choukrallah and Markhart, unpublished data; Smit et al., 1989; Gollan et al., 1986).

Quantifying water, nutrient, and plant growth substance transport from the root to the shoot is fundamental to understanding how plants cope with a wide variety of environmental insults. The ability of the root. system to meet transpirational demands of the shoot is determined by root -hydraulic conductance. Root hydraulic conductance is a function of the specific hydraulic conductivity of a given unit of root, the relationship between absorbing and nonabsorbing fractions of the root, and the total root biomass. Techniques for measuring root hydraulic conductivity are relatively simple, but not necessarily easy. We have tried here to provide an understanding of fundamental root processes and describe the techniques and precautions necessary to generate meaningful estimates of root hydraulic conductivity. We also recognize that understanding just the flux of water through root systems is not sufficient to understand how the root affects the shoot. The corollary is also true. Understanding just the flux of nutrients and plant growth substances is also insufficient.

The future holds much promise. As techniques for analyses of plant growth substances become more readily available and decrease in cost, the analysis of water flux can be coupled with the quantification of plant growth substance flux and concentration. This information will greatly increase our understanding of how roots react to their environment and how they affect the growth and development of the shoot.

\section{Literature Cited}

Andersen, C.P., A.H. Markhart, III, R.K. Dixon, and E.I. Sucoff. 1988. Root hydraulic conductivity of vesicular-arbuscular mycorrhizal green ash seedlings. New Phytol. 109:465-471.

Anderson, W.P., D.P. Aikman, and A. Meiri. 1970. Excised root exudation-A standing gradient osmotic flow. Proc. R. Soc. London, Ser. B. 174:445-458.

Baker, D.A. and D.R. Lachno. 1987. Osmotic and salt stress induction of ABA in excised maize roots. Third Intl. Symp. on Structure and Function of Roots. Nitra, Czechoslovakia.

Bowling, D.J.F., B.T. Watson, and R. Ehwald. 1985. The effect of phloem ringing on root growth and potassium uptake by Helianthus annuus. J. Expt. Bot. 36:290-297.

Dalton, F.N., P.A.C. Raats, and W.R. Gardner. 1975. Simultaneous uptake of water and solutes by plants. Agron. J. 67:334-339.

Davies, W.J., J. Metcalfe, T.A. Lodge, and A.R. Da Costa. 1986. Plant growth substances and the regulation of growth under drought. Austral. J. Plant Physiol. 13:105-125.

DeBoer, A.H. and H.B.A. Prins. 1984. Trans-root electrical potential in roots of Plantago media L. as affected by hydrostatic pressure: The induction of an $\mathrm{O}_{2}$ deficient core. Plant \& Cell Physiol. 25:643-655.

Fiscus, E.L. 1975. The interaction between osmotic- and pressure-induced water flow in plant roots. Plant Physiol. 55:917-922.

Fiscus, E.L. 1986. Diurnal changes in volume and solute transport coefficients of Phaseolus roots. Plant Physiol. 80:752-759.

Fiscus E.L. and A.H. Markhart, III. 1979. Relationships between root system water transport properties and plant size in Phaseolus. Plant Physiol. 64:770-773.

Gollan, T., J.B. Passioura, and R. Munns. 1986. Soil water status affects the stomatal conductance of fully turgid wheat and sunflower leaves. Austral. J. Plant Physiol. 13:145-154.

Hanson, P.J., E.I. Sucoff, and A.H. Markhart, III. 1985. Quantifying apoplastic flux through red pine root systems using trisodium, 3-hydroxy5,8,10- pyrenetrisulfonate, Plant Physiol. 77:21-24.

Klepper, B. 1967. Effects of osmotic pressure on exudation from corn roots. Austral. J. Biol. Sci. 20:723-735.

Kramer, P.J. 1983. Water relations of plants. Academic, New York.

Lauchli, A. 1976. Symplastic transport and ion release to the xylem, p. 101-112. In: I.F. Wardlaw and J.B. Passioura (eds.). Transport and trans- 
fer processes in plants.

Markhart, A.H., III. 1978. The coupled uptake of solute and solvent by plant root systems under simulated high transpiration rates. PhD Diss., Duke Univ. Durham, N.C.

Markhart, A.H., III. 1987. Chilling injury: A review of possible causes. HortScience 22:1329-1333.

Markhart, A.H., III, E.L. Fiscus, A.W. Naylor, and P.J. Kramer. 1979. Effect of temperature on water and ion transport in soybean and broccoli root systems. Plant Physiol. 64:83-87.

Markhart, A.H., III, M.M. Peet, N. Sionit, and P.J. Kramer. 1980. Low temperature acclimation on root fatty acid composition, leaf water potential, gas exchange and growth of soybean seedlings. Plant Cell Environ. 3:435-441.

Newman, E.I. 1976. Water movement through root systems. Phil. Trans. R. Soc. London, Ser. B. 273:463-478.

O'Leary, J.W. 1969. The effect of salinity on the permeability of roots to water. Israel J. Bot. 18:1-9.

Parsons, L.R. and P.J. Kramer. 1974. Diurnal cycling in root resistance to water movement. Physiol. Plant. 30:19-23.

Passioura, J.B. 1988. Water transport in and to roots. Annu. Rev. Plant Physiol. 39:245-265.

Peterson, C. 1988. Hypodermal layers in roots. What's new in plant phys- iology. Physiol. Plant. 1:204-208.

Salim, M. and M.G. Pitman. 1984. Pressure-induced water and solute flow through plant roots. J. Expt. Bot. 35:869-881

Shalhevet, J., E.V. Mass, G.D. Hoffman, and G. Ogata. 1976. Salinity and the hydraulic conductance of roots. Physiol. Plant. 38:224-232.

Smit, B. and M. Stachowiak. 1988. The effects of hypoxia and elevated carbon dioxide levels on water flux through Populus roots. Tree Physiol. 4:153-165.

Smit, B., M. Stachowiak, and E. Van Volkenburgh. 1989. Cellular processes limiting leaf growth in plants under hypoxic root stress. J. Expt. Bot. 40:69-94.

Smit-Spinks, B., B.T. Swanson, and A.H. Markhart, III. 1984. Changes in water relations, water flux and root exudate abscisic content with cold acclimation of Pinus sylvestris. Austral. J. Plant Physiol. 11:431-441.

Steudle, E. and W.D. Jeschke. 1983. Water transport in barley roots. Planta 158:237-248.

Teskey, R.O., T.M. Hinkley, and C.C. Grier. 1984. Temperature induced change in water relations of Abies amabilis (Douge) Forbes. Plant Physiol. 74:77-80.

Walton, D.C., M.A. Harrison, and P. Cote. 1976. The effects of water stress on abscisic acid levels and metabolism in roots of Phaseolus vulgaris and other plants. Planta 131:141-144. 